\title{
Visuelle Aspekte von Modellen
}

Visuelle Modelle sind anschaulich, weil sie zeigen, was sie uns erklären. Man kann gewissermaßen sehen, was das Modell uns sagt. Dieses visuelle Verständnis von Modellen möchte ich mit Hilfe von Wittgensteins Begriff des "Aspektsehens" näher untersuchen. Einen Aspekt zu sehen bedeutet für Wittgenstein, einen Gegenstand als etwas zu sehen. Der Hasen-Enten-Kopfkann zum Beispiel als Hase oder als Ente gesehen werden. Gleichzeitig verschiebt sich dabei der begriffliche Rahmen, mit dem wir das Gesehene beschreiben. Indem wir ein Modell als Modell sehen, lernen wir, es auf eine neue Weise und mit neuen Begriffen zu beschreiben. Das visuelle Verständnis, das uns Modelle vermitteln, ist folglich keine Illustration einer sprachlichen Erklärung, sondern die neu gewonnene Fähigkeit, Dinge auf eine bestimmte Weise zu sehen und $z$ u beschreiben.

Mit Modellen werden komplizierte Zusammenhänge anschaulich gemacht. Dabei ist der Ausdruck »anschaulich" bei den meisten Modellen ganz wörtlich zu verstehen: Man kann sie anschauen, sei es in materieller Form oder zumindest vor dem inneren Auge. Visuelle Modelle, um die es im Folgenden gehen soll, sind also Gegenstände, die visuell erfahrbar sind oder es zumindest sein könnten. Sie lassen sich betrachten, sie werden gezeigt, vorgeführt oder abgebildet. Das Bohrsche Atommodell, das Lehr-Modell einer Dampfmaschine, das Computermodell eines Luftwirbels oder das Architekturmodell eines Gebäudes sind Beispiele solcher Modelle. Ihre Anschaulichkeit spielt eine wichtige Rolle für ihre Funktionsweise als Modell.

Die Anschaulichkeit von Modellen, das heißt die Tatsache, dass man sie sehen kann, ist natürlich kein Selbstzweck. Wir betrachten Modelle nicht um ihrer selbst willen, sondern mit dem Ziel, etwas anderes zu verstehen, sei es die Funktionalität einer Maschine oder der Aufbau eines zukünftigen Gebäudekomplexes. Modelle sind immer Modelle von und für solche anderen Dinge, ${ }^{1}$ das heißt, sie stehen

1 Bernd Mahr: »Modellieren - Beobachtungen und Gedanken zur Geschichte des Modellbegriffs«. In: Sybille Krämer, Horst Bredekamp (Hg.): Bild - Schrift - Zahl, München 2003, S. 67. Gewöhnlich wird der Modellbegriff in Anlehnung an die Semiotik triadisch verstanden: eine Person nimmt etwas als Modell für etwas anderes (Marks W. Wartofsky: Models. Representation and the Scientific Understanding, Dordrecht 1997). Mahr spaltet die Beziehung zwischen Modell und Modelliertem weiter auf: Modelle sind Modelle von etwas und für etwas, das heißt Modelle als Bestimmung von etwas Bestehendem und als Vorbild für etwas zu Schaffendes. Aber auch diese Unterscheidung darf nicht darüber hinwegtäuschen, dass jede Charakterisierung von Modellen, bei der auf etwas Anderes, dem Modell Externes verwiesen wird, immer problematisch ist. Denn häufig wird dieses Andere erst 
im Verhältnis eines Ab- oder Vorbildes zu den Dingen, die sie beschreiben und erklären. Wenn wir das Modell einer Dampfmaschine betrachten, so wollen wir eben die Dampfmaschine verstehen und nicht (nur) das Modell davon.

Die Anschaulichkeit spielt dabei eine zentrale Rolle. Denn visuelle Modelle zeigen, was sie erklären. Sie zeigen uns Zusammenhänge, Abläufe, Funktionen oder Proportionen, und das Zeigen besteht darin, dass diese Zusammenhänge, Abläufe, Funktionen oder Proportionen am Modell selbst sichtbar werden. Modelle verweisen also nicht nur auf andere Dinge, von denen sie Modell sind, sondern sie verweisen immer auch auf sich selbst, als Träger von Eigenschaften, die auch dem zu erklärenden Gegenstand zugeschrieben werden. Das Architekturmodell zeigt uns die Proportionen eines zukünftigen Gebäudes, indem es diese Proportionen selbst besitzt. Und das Modell der Dampfmaschine zeigt uns die Funktionsweise einer Dampfmaschine, indem es in idealisierter Form ${ }^{2}$ diese Funktionsweise ebenfalls besitzt oder zumindest nach vergleichbaren Regeln funktioniert. Die Aussage eines visuellen Modells in Bezug auf einen modellierten Gegenstand lautet: "So funktioniert das, so sieht das aus, so ist der Zusammenhang $\mathrm{zu}$ verstehen."

Modelle, kann man also sagen, machen Zusammenhänge anschaulich, indem diese Zusammenhänge am Modell selbst gesehen werden können. Für die Wahrnehmung von Modellen scheint zu gelten, dass die relevanten, das heißt übertragbaren Eigenschaften dabei visuell identifiziert werden müssen. Man betrachtet das Modell, sieht bestimmte Zusammenhänge und folgert, dass man die gleichen Zusammenhänge bei den Dingen findet, von denen oder für die das Modell ein Modell ist. Das entspricht der Standardauffassung nicht nur von visuellen Modellen, sondern von Modellen im Allgemeinen. Danach sind Modelle, seien sie nun anschaulich oder nicht, eine Art von Repräsentation, die Strukturen mit dem Repräsentierten gemeinsam haben - wobei diese Strukturen meist in idealisierter oder abstrahierter Form am Modell vorhanden sind. ${ }^{3}$ Ein Modell zu verstehen ist ein Vorgang, der in zwei Schritte unterteilt werden kann: Zuerst werden bestimmte Eigenschaften oder Strukturen am Modell identifiziert und anschließend werden sie in einem Interpretationsschritt mit den »externen« modellierten Dinge in einen Zusammenhang gebracht.

Ich möchte im Folgenden zeigen, dass diese Auffassung problematisch ist, insbesondere bei visuellen Modellen. Ihre Wahrnehmung kann nicht von dem

durch das Modell geschaffen. Nirgends ist diese Abhängigkeit so deutlich zu sehen wie in den Naturwissenschaften, deren Modelle gleichzeitig mit einer modellhaften Wirklichkeit identifiziert werden. Das Atommodell ist ein Modell von Atomen, aber diese Atome sind selbst nichts anderes als wiederum diese modellhafte Vorstellung davon. Das Modell und das, wovon es ein Modell ist, fallen zusammen.

2 Idealisierung ist eine der Haupteigenschaften von Modellen, was in wissenschaftlicher Hinsicht jedoch nicht unproblematisch ist. Siehe Ernan McMullin: „Galilean Idealization«. In: Studies in the History and Philosophy of Science 16 (1985), S. 247-273.

3 Siehe zum Beispiel Elisabeth A. Lloyd: »Models«. In: Routledge Encyclopedia of Philosophy, London 1998, Bd. 6, S. 443-447. 


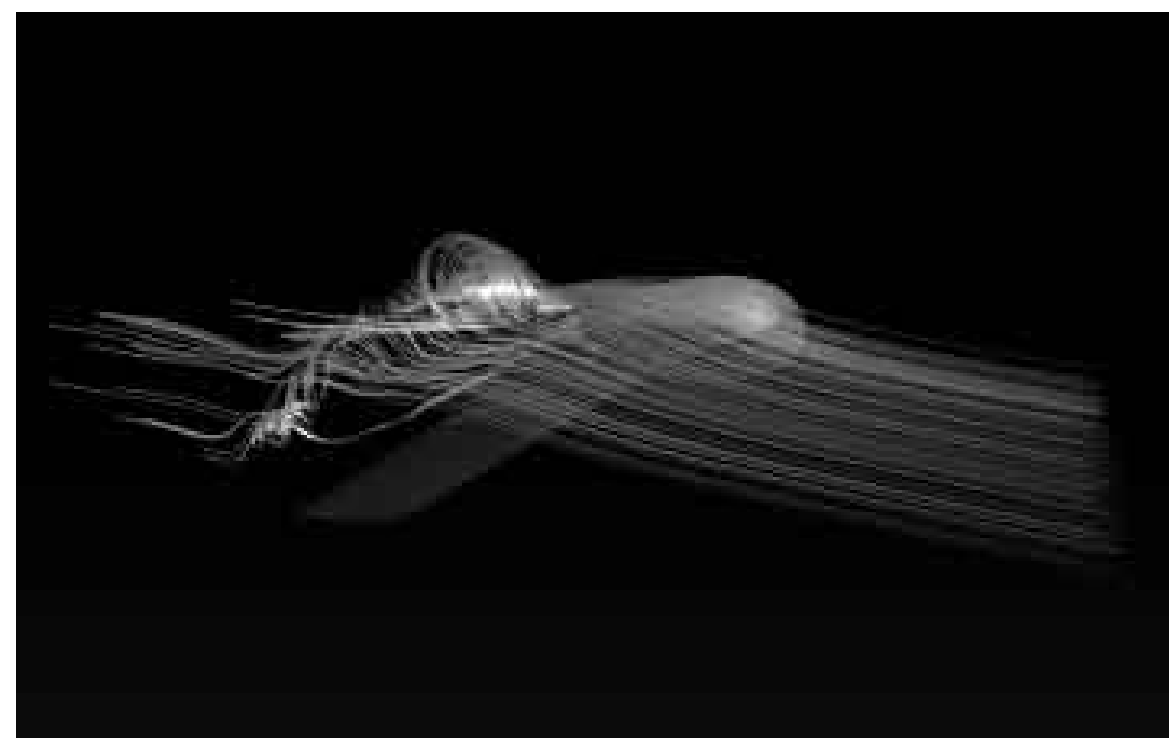

Abb. 1: Strömung um einen Flugzeugflügel. Simulation: Frank Thiele, TU Berlin. Visualisierung: Tino Weinkauf, ZIB. Siehe auch Farbtafel I.

Interpretationsprozess getrennt werden, bei dem der modellierte Gegenstand ins Spiel kommt. Die Interpretation des Modells als Modell von etwas Externem ist kein getrennter zweiter Schritt, sondern stattdessen Teil der visuellen Erfahrung. Visuelle Modelle, so lautet die These, werden als Modelle von etwas wahrgenommen, das heißt, sie werden nicht "unvoreingenommen" hinsichtlich der Dinge gesehen, für die sie ein Modell sind. Mein Ziel ist es zu zeigen, dass die Anschaulichkeit von Modellen als ein Ineinandergreifen von visueller Wahrnehmung und Interpretation verstanden werden muss. Sehen und Verstehen bilden eine Einheit, die weit über einen illustrativen Charakter hinausgeht. Modelle illustrieren keine Erklärung, sondern geben eine visuelle Erklärung. Die gewonnene Erkenntnis ist dabei selbst visueller Natur und kann nicht durch andere Erklärungsformen ersetzt werden.

II

Abbildung 1 zeigt das vom Computer berechnete Modell eines Luftwirbels, der sich an einem Flügel bildet. Reale Luftwirbel sind Strömungen im Raum, die komplexe Formen annehmen können. Wirbel sind in Wirklichkeit nicht sichtbar, was nicht nur daran liegt, dass die Luft, in der der Wirbel sich bildet, unsichtbar ist. Auch in farbigen Gasen könnte man einen Wirbel nicht gut sehen. Wirbel sind keine materiellen Objekte im gewöhnlichen Sinn, sondern sind durch eine Bewegung beziehungsweise durch einen Strömungsverlauf bestimmt. Ganz abgesehen 
davon haben sie auch keine klar abgegrenzte Form, das heißt, es gibt keine klare Unterscheidung zwischen den Gebieten, die zum Wirbel dazugehören, und denen, die außerhalb von ihm liegen. Luftwirbel haben weder eine fest umrissene Gestalt noch gibt es eine eindeutig definierbare Oberfläche, die man sehen oder einfärben könnte.

Trotzdem handelt es sich bei der Abbildung um ein visuelles Modell eines Wirbels. Das Computermodell zeigt uns den Wirbel auf anschauliche Weise. Und die oben beschriebene Auffassung von Modellen würde auch hier eine Erklärung anbieten: Das vom Computer gerechnete Modell besteht aus leuchtenden Linien, sogenannten »Illuminated Field Lines ${ }^{4}{ }^{4}$ Dabei werden Luftsegmente, die nebeneinander in einer Reihe liegen, bei ihrer Bewegung durch den Wirbel als leuchtende Linien dargestellt. Die Linien, die wir auf dem Bild sehen, entsprechen in idealisierter, simulierter Form den realen Bewegungslinien von Luftsegmenten. Aus der Form der einzelnen Linien kann man also Rückschlüsse auf genau definierte Eigenschaften eines Wirbels ziehen. Zusammenhänge, die am Modell sichtbar sind, können so auf reale oder mögliche Objekte übertragen werden. Doch macht dies die Anschaulichkeit des Modells aus? Wird uns der Wirbel anschaulich gemacht, indem wir eine solche Übertragung leisten können? Sicherlich sieht man die Form der einzelnen Linien, aus denen das Modell besteht. Aber was das Computermodell anschaulich macht, scheinen nicht so sehr diese Linien zu sein, die wir sehen, sondern der Wirbel, der in den Linien auf eine Weise gesehen werden kann; oder man könnte auch sagen: Wir sehen die Linien als Wirbel. Es sind keine "neutralen" Linien, die wir wahrnehmen und dann ihre Eigenschaften identifizieren, sondern schon in der Wahrnehmung wird das Gesehene als Wirbel interpretiert.

Doch was bedeutet das genau? Zum Beispiel verbindet unser Auge die einzelnen Linien zu übergeordneten Strukturen. Mehrere nebeneinander liegende Linien sehen wir als Fläche, deren Zwischenraum eindeutig ausgefüllt werden könnte. Dies ist keine triviale Leistung des Betrachters, denn es gelingt uns nicht bei jeder Ansammlung von Linien, sondern nur bei solchen, wo eine gewisse Stetigkeit erfüllt ist. Das wird an den "ungeordneten « Ausschnitten des Modell-Wirbels deutlich, wo uns dieser visuelle Zusammenhang verloren geht: Wir sehen nur noch ein Knäuel von Strichen. Die Auswahl der Linien, aus denen das Modell gezeichnet wird, spielt also eine große Rolle dabei, ob wir solche übergeordneten Formen visuell erkennen können. Bestünde das Modell aus zu vielen Linien in mehreren Schichten, so wären wir schnell visuell überfordert und könnten keine Struktur mehr sehen. Das Gleiche würde gelten, wenn es zu wenige Linien gäbe, deren Zusammenhang für uns nicht mehr sichtbar ist. Wir erkennen die übergeordneten Strukturen, indem wir diese sehen. Wir können die einzelnen Linien wie

4 Detlev Stalling, Malte Zöckler, Hans-Christian Hege: „Fast Display of Illuminated Field Lines«. In: IEEE Transactions on Visualization and Computer Graphics 3.2 (1997), S. 118-128. 
selbstverständlich miteinander verbinden und können sie als ein Ganzes beschreiben und auch visuell wiedererkennen. Man könnte sogar so weit gehen und behaupten, dass wir nur diese übergeordneten Strukturen sehen und gar nicht mehr die Einzellinien, aus der sie aufgebaut ist. Denn im Gegensatz zu dem gesamten Wirbel, den wir auf den ersten Blick beschreiben und wiedererkennen können, müssen wir den Verlauf einer einzelnen Linie erst mühevoll mit dem Finger nachverfolgen, um das Gleiche zu tun.

Das Modell wird erst dadurch anschaulich, dass wir eine zusammenhängende Form in den Einzellinien sehen können. Und das macht die Leistung von guten visuellen Modellen ja aus: Sie finden eine Darstellungsweise, die übergeordnete Aspekte sichtbar macht, die sonst im Gewimmel der Details verborgen blieben. Die Anschaulichkeit der »Illuminated Field Lines« beruht also nicht darauf, dass wir die einzelnen Linien sehen können, sondern dass wir diese visuell zu einem Gesamtbild formen. Und die visuelle Interpretation geht darüber noch hinaus. Wir sehen nicht nur eine übergeordnete Struktur in den Linien, sondern bringen diese auch in einen Bedeutungszusammenhang, hier mit einem realen Wirbel. Denn die übergeordnete Struktur und auch den Linienverlauf sehen wir nicht als "starr", sondern sehen auf eine Weise eine Bewegungsrichtung in ihn hinein. Wir »sehen" die Linien von rechts nach links verlaufen, und wir »sehen", wie sie vom Flügel abgelenkt und verwirbelt werden, sobald wir den Flügel als solchen erkennen. Die leuchtenden Linien als einen Wirbel zu sehen, ist in etwa so, wie zwei Punkte und einen Strich als ein Gesicht zu sehen. ${ }^{5}$ Visuelle Wahrnehmung und Interpretation greifen ineinander und sind miteinander verbunden. Wer die Linien einmal als das Modell des Wirbels gesehen hat, für den wäre es nicht mehr leicht, sie zum Beispiel als Haarlocke zu sehen, ${ }^{6}$ wie es ebenso schwer wäre, ein Strichgesicht nicht als Gesicht zu sehen. In der Wahrnehmung des visuellen Modells »spiegelt« sich dessen Interpretation wider.

\section{III}

Die Aussagen des letzten Absatzes sind voller Anführungszeichen. Auf eine Weise kann man eine übergeordnete Struktur oder auch eine Bewegungsrichtung "sehen " oder "hineinsehen", aber es scheint doch vermessen, dies als ein direktes Sehen zu bezeichnen. Das »Widerspiegeln« der Interpretation im Modell scheint weder eine rein gedankliche Interpretation noch eine reine visuelle Wahrnehmung

5 Eine sehr ähnliche Bemerkung findet sich bei Gottfried Boehm: „Ikonisches Wissen. Das Bild als Modell«. In: ders.: Wie Bilder Sinn erzeugen. Die Macht des Zeigens, Berlin 2007, S. 114-140.

6 Interessant ist dabei der visuelle Effekt, der entsteht, wenn man das Bild herumdreht. Die Linien können nicht mehr so gut in den Bedeutungszusammenhang gestellt werden und so sehen wir sie auch nicht mehr als einen Wirbel, der zum Beispiel eine klare Bewegungsrichtung hat, sondern viel eher als eine starre Form. 
zu sein. Ich möchte diese Fragen in Anlehnung an Wittgensteins sehr spezielle, aber auch ausführliche Diskussion über das Verhältnis von visueller Wahrnehmung und dem Verständnis des Wahrgenommenen untersuchen.

Das Phänomen, das Wittgenstein interessiert, ist die Wahrnehmung von $A s$ pekten, wie er es nennt, oder im visuellen Fall von "sehen als «. ${ }^{7}$ Man kann einen Gegenstand als etwas sehen, das eine Bedeutung hat. Wittgensteins besonderes Interesse an diesem Phänomen muss man vor dem Hintergrund seiner Auseinandersetzung mit Sprache und Verstehen sehen. Für Wittgenstein ist die Bedeutung sprachlicher Ausdrücke durch ihren Gebrauch gegeben, den wir in "Sprachspielen" von ihnen machen. Man erklärt die Bedeutung von einem sprachlichen Ausdruck (zum Beispiel »Hilfe!« oder »Heute regnet es nicht«), indem man ihren Gebrauch oder ihre Verwendung erklärt. Der Gebrauch wiederum richtet sich nach Regeln und somit wird die Bedeutung eines Ausdrucks erklärt, indem man die Regeln angibt, nach denen er verwendet werden soll. Das Verstehen von Bedeutung ist das Verstehen eines bestimmten Gebrauchs des Bedeutungsträgers und somit die Beherrschung von Regeln und ihrer Anwendung. Etwas zu verstehen ist nichts anderes als die Fähigkeit, richtig zu handeln.

Dieses Konzept von Verstehen als Fähigkeit sieht Wittgenstein vor allem im Gegensatz zum Konzept von Verstehen als innerer Vorgang oder als ein Erlebnis. Das Erlebnis, das jemand hat, wenn er einen Satz versteht, kann laut Wittgenstein kein Kriterium dafür sein, dass er etwas verstanden hat. Wer einen Satz versteht und sich danach richtet, der tut das "direkt « und könnte die Erlebnisse dabei meist gar nicht beschreiben. Zwischen einer Regel und ihrer Anwendung muss kein inneres Erlebnis vermittelt sein. »Ich folge der Regel blind « lautet Wittgensteins bekanntes Diktum. ${ }^{8}$

7 Die Auseinandersetzung reicht zurück bis in die Philosophie des Tractatus und zieht sich durch die gesamte Philosophie Wittgensteins. Die wichtigsten Stellen sind Tractatus 5.5423; Braunes Buch $\$ 137 \mathrm{ff}$., II $₫ 16 \mathrm{ff}$., II $₫ 23$; Philosophische Grammatik $₫ 3 \mathrm{ff} ., \$ 123-132$; Zettel \$162-188 und viele Stellen in den Bemerkungen über die Philosophie der Psychologie und Philosophische Untersuchungen II, S. 518-578.

Die ausführlichste Darstellung findet sich dabei im zweiten Teil der Philosophischen Untersuchungen, wo er sich auch mit anderen "psychologischen « Begriffen auseinandersetzt. Er will zeigen, dass Begriffe wie Verstehen, Absicht, Hoffnung, Meinen, Glauben et cetera keine inneren Erlebnisse oder Vorgänge benennen. Seine "Vorstudien« und Tagebucheinträge dazu wurden daher unter dem Titel »Philosophie der Psychologie« herausgegeben. (Siehe zu diesem Begriff PUII S. 580 und PUII S. 497-499).

Ich werde für die Texte von Wittgenstein folgende Abkürzungen verwenden:

BPP1: Ludwig Wittgenstein, Bemerkungen zur Philosophie der Psychologie, Bd. 1.

BPP2: Ludwig Wittgenstein, Bemerkungen zur Philosophie der Psychologie, Bd. 2.

BPP3: Ludwig Wittgenstein, Letzte Schriften über die Philosophie der Psychologie.

PU: Ludwig Wittgenstein, Philosophische Untersuchungen.

PUII: Ludwig Wittgenstein, Philosophische Untersuchungen, Teil 2.

Alles in: Ludwig Wittgenstein, Werkausgabe, Frankfurt am Main 1984.

8 PU $\$ 219$. 

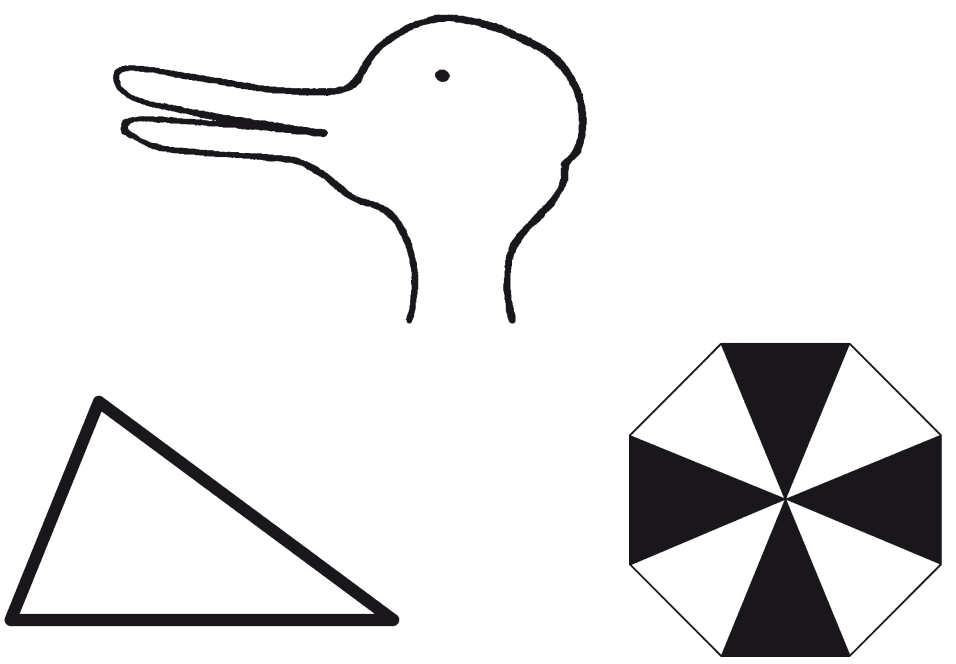

Abb. 2-4: Hasen-Enten-Kopf, Dreieck, Doppelkreuz.

Die Wahrnehmung von Aspekten stellt für Wittgenstein nun eine Herausforderung dar, weil sie dieser These zu widersprechen scheint. Beim Sehen von etwas als etwas wirkt sich eine bestimmte Interpretation, also ein bestimmtes Verständnis des Gesehenen, auf die Wahrnehmungserlebnisse aus. Der doppeldeutige HasenEnten-Kopf (Abb. 2) ist das bekannteste Beispiel dafür. Man kann ihn als Bild eines Hasen oder als Bild einer Ente interpretieren, und man sieht die Zeichnung dann jeweils anders. Das Sehen fällt hier mit dem Verstehen zusammen: Wer die Zeichnung als Hasenbild sieht, der versteht sie auch als etwas, das einen Hasen darstellt. Wittgenstein diskutiert eine ganze Reihe solcher Aspektwahrnehmungen. Man kann ein Dreieck in verschiedenen Aspekten sehen, nämlich als Pfeil, der in verschiedene Richtungen zeigt (Abb. 3). ${ }^{9}$ Ein weiteres Beispiel ist die Ähnlichkeit zwischen zwei Gesichtern, die man bisher nicht bemerkt hat und die man plötzlich aufleuchten sieht. ${ }^{10}$ Man sieht das eine oder die beiden Gesichter plötzlich anders, nämlich als ähnlich. Auch ein "Doppelkreuz« (Abb. 4) kann zwei verschiedene Aspekte haben, so dass es einmal als ein schwarzes Kreuz auf weißem Grund und einmal als weißes Kreuz auf schwarzem Grund gesehen wird. ${ }^{11}$ Zuletzt gibt es Vexierbilder, also Bilder, auf denen ein Gegenstand versteckt ist, die man lange betrachten muss, bis plötzlich der gesuchte Gegenstand visuell erscheint.

9 PUII S. 530b.

10 PUII S. 518a.

11 PUII S. 541d. 
Was ist nun das Gemeinsame an diesen Fällen? Wittgenstein beginnt seine Diskussion mit einem Beispiel, bei dem eine Person plötzlich eine Ähnlichkeit zwischen zwei Gesichtern bemerkt:

Zwei Verwendungen des Wortes "sehen«.

Die eine: "Was siehst du dort? - " Ich sehe dies" (es folgt eine Beschreibung, eine Zeichnung, eine Kopie). Die andere: »Ich sehe eine Ähnlichkeit in diesen beiden Gesichtern « - der, dem ich dies mitteile, mag die Gesichter so deutlich sehen wie ich selbst.

Die Wichtigkeit: Der kategorische Unterschied der beiden `Objekter des Sehens.

Der Eine könnte die beiden Gesichter genau abzeichnen; der Andere in dieser Zeichnung die Ähnlichkeit bemerken, die der erste nicht sah.

Ich betrachte ein Gesicht, auf einmal bemerke ich seine Ähnlichkeit mit einem anderen. Ich sehe, daß es sich nicht geändert hat; und sehe es doch anders. Diese Erfahrung nenne ich "das Bemerken eines Aspektes".

Seine Ursachen interessieren den Psychologen.

Uns interessiert der Begriff und seine Stellung in den Erfahrungsbegriffen. ${ }^{12}$

Worin besteht also der »kategorische Unterschied « zwischen den beiden »Objekten" des Sehens, auf den sich Wittgenstein hier bezieht? Wer einen Gegenstand sieht, der kann bestimmte Eigenschaften von ihm erkennen und eine Beschreibung davon geben. Eine solche Beschreibung kann zutreffen oder nicht und ob sie das tut, ist eine "empirische" Frage. Es wäre möglich, dass der beschriebene Gegenstand die Eigenschaft nicht besitzt, die man ihm zuschreibt, oder zu einem anderen Zeitpunkt nicht mehr besitzt. Wittgenstein spricht von "zeitlichen « Sätzen oder "Erfahrungssätzen «. ${ }^{13}$ Davon müssen Sätze unterschieden werden, die einen begrifflichen oder auch logischen Zusammenhang ausdrücken, der auf Grund von Regeln ${ }^{14}$ gegeben ist. Aussagen wie »Wenn Paul ein Junggeselle ist, dann ist er ein unverheirateter Mann. "oder "Das Standardmeter in Paris ist $1 \mathrm{~m}$ lang." sind keine Erfahrungssätze über die Eigenschaften von Gegenständen, sondern sie betreffen die Regeln, nach denen bestimmte Begriffe verwendet werden beziehungsweise verwendet werden sollen. Wittgenstein nennt sie "grammatische", "zeitlose» oder "geometrische« Sätze ${ }^{15}$, die eine "interne

12 PUII S. 518.

13 Siehe zum Beispiel BPP3 $\$ 152$ und $\$ 759$.

14 Wittgenstein spricht im Zusammenhang von Aspektwahrnehmung nur sehr selten von Regeln, obwohl mir dies zum besseren Verständnis seiner Position unerlässlich erscheint. Begriffe wie »Deutung ", "Auffassung", "Verwendungsweise«, "interne Relation« oder "zeitloser Satz«, mit deren Hilfe er Aspektwahrnehmung diskutiert, hängen direkt mit seinem Verständnis von Regeln zusammen. Wittgenstein vermeidet den Begriff, so scheint mir, weil bei den meisten Verwendungen sich die Regeln nicht klar genug formulieren lassen. Siehe zum Beispiel BPP3 $\$ 968$ oder BPP1 $\$ 588$.

15 Siehe beispielsweise BPP3 $\$ 146$ und $\$ 152$. 
Relation ${ }^{16}{ }^{6}$ bezeichnen. Die Aussage »Paul ist ein unverheirateter Mann « und die Aussage »Paul ist ein Junggeselle« sind zeitlos miteinander verbunden. Man kann sich also keine Situation vorstellen, in der die eine Aussage zutrifft und die andere nicht. Ebenso steht der Sachverhalt, dass X ein Junggeselle ist, in einer internen Relation zu dem Sachverhalt, dass $X$ unverheiratet ist. Es ist keine empirische Frage, ob die beiden Sachverhalte immer zusammen aufzufinden sind, sondern es gibt eine begriffliche Regel, dass sie das tun müssen. Man kann also unterscheiden zwischen empirischen Zusammenhängen einerseits und begrifflichen, logischen, normativen oder eben »internen « Zusammenhängen andererseits.

Die Ähnlichkeit von zwei Gegenständen steht nun in einer internen Relation zu den entsprechenden Eigenschaften dieser Gegenstände. Denn ähnlich nennt man zwei Gegenstände genau dann, wenn sie in manchen ihrer Eigenschaften übereinstimmen. Gibt es eine gemeinsame Eigenschaft, zum Beispiel eine gemeinsame Farbe, so sind zwei Gegenstände auch in dieser Hinsicht ähnlich - um das herauszufinden, müssen wir keine empirische Forschung betreiben, sondern nur den Begriff der Ähnlichkeit beherrschen. Der erste Betrachter in Wittgensteins Beispiel, der die Ähnlichkeit zwischen den beiden Gesichtern bemerkt, sieht also einen Zusammenhang zwischen den beiden Gesichtern, der sich für ihn begrifflich ergibt, wenn er die zu Grunde liegenden Eigenschaften schon zuvor gesehen hat. Nehmen wir einmal an, er kann sehen, dass das erste Gesicht eine Hakennase hat. Er kann also die Beschreibung geben: "Das Gesicht A hat eine Hakennase«. Das Gleiche gelte für das zweite Gesicht, das er mit "Das Gesicht B hat eine Hakennase» beschreiben könnte. Daraus folgt, dass die beiden Gesichter A und B in dieser Hinsicht einander ähnlich sind. Oder anders formuliert: Die beiden Beschreibungssätze "Das Gesicht A hat eine Hakennase» und "Das Gesicht B hat eine Hakennase " sind auf Grund einer begrifflichen Regel mit dem Satz "A und B sind ähnlich" verbunden. Die Aussage: "Wenn das Gesicht A und das Gesicht B hakennasig sind, so sind sie auch ähnlich«, ist ein "zeitlos« wahrer Satz. Wer einen solchen Zusammenhang plötzlich bemerkt, obwohl er die zu Grunde liegenden Eigenschaften schon zuvor gesehen hat, der macht eine logische Entdeckung. Die Erkenntnis, die er gewinnt, ist "von der Art mathematischer Entdeckungen", wie Wittgenstein schreibt. ${ }^{17}$ "Wenn die Gesichter beide $s o$ aussehen, dann sind sie ja auch ähnlich!« könnte der Betrachter sagen und diese Aussage lässt sich vergleichen mit »Wenn die Äpfel 5 kg wiegen, so wiegen sie ja auch 10 Pfund!«.

16 Der Begriff stammt aus einer Diskussion im Zusammenhang mit dem Idealismus und bezeichnete dort Eigenschaften, die ein Gegenstand notwendigerweise hat. Im Tractatus spielt der Begriff eine wichtige Rolle. Wittgenstein fasst interne Relationen dabei als logische Relationen auf, die nicht von einem Gegenstand gesagt, sondern nur in der Struktur der entsprechenden Sätze gezeigt werden können (Tractatus 4.123 ff.). In seiner späteren Philosophie gehören interne Relationen, wie alle logischen Beziehungen, zur Grammatik, also zu den Regeln eines Gebrauchs. Eine gute Darstellung findet sich bei Hans-Johann Glock: A Wittgenstein Dictionary, Oxford 1996, S. 189 ff.

17 Siehe zum Beispiel BPP2 $\$ 438 \mathrm{f}$. 
Dieser »mathematische« Charakter des Bemerkens von Ähnlichkeiten ist nicht abhängig davon, dass der Betrachter die zu Grunde liegende Eigenschaft vorher sprachlich formuliert hat. Wer eine Ähnlichkeit visuell bemerkt, der muss immer auch die zu Grunde liegenden Eigenschaften sehen, auch wenn er sie vielleicht nicht sprachlich beschreiben kann. Wenn der Betrachter aber diese Eigenschaften sieht, dann ist die Ähnlichkeit selbst etwas, das logisch daraus folgt. Er sieht nicht etwas Neues in dem Sinne, dass sich die Gesichter verändern, sondern in dem Sinne, dass er einen Zusammenhang bemerkt, der auf den gesehenen Gesichtern aufbaut. Der "kategorische Unterschied der beiden Objekte", die in Wittgensteins Beispiel gesehen werden, ist also der folgende: Im ersten Fall sieht der Betrachter einen "empirischen « Sachverhalt, der am besten durch Sätze beschrieben wird, die empirisch wahr oder falsch sind. Im zweiten Fall aber sieht der Betrachter eine interne Relation, die durch Sätze beschrieben wird, die auf Grund von einem logisch begrifflichen Zusammenhang wahr oder falsch sind. ${ }^{18}$

Dies findet man auch in den anderen Beispielen von Aspektwahrnehmung. Besonders deutlich wird das am Beispiel von Vexierbildern, also von Zeichnungen, in denen eine bestimmte Form versteckt ist. Nehmen wir an, die gesuchte Form sei eine einfache Zickzack-Linie, die gut in den umgebenden Linien verborgen ist. Wenn man sie plötzlich in der Zeichnung erkennt, so bemerkt man einen »internen« Zusammenhang zwischen der Zeichnung und der Form. Denn schon zuvor hat man alle Details der Zeichnung klar und deutlich gesehen. Man hätte also eine Beschreibung des Bildes geben können durch eine genaue Angabe über die Verteilung der Striche oder auch, indem man eine Kopie der Zeichnung gibt. Und in dieser Beschreibung wäre die Zickzacklinie dann »logisch « schon enthalten gewesen. Man hätte aus der Beschreibung ableiten können, dass sich die gesuchte Linie in der Zeichnung verbirgt.

Wenn ich sage: "Schau! in diesem Bild ist diese Figur enthalten « - mache ich eine geometrische Bemerkung? - Ist >dieses Bild ‘ nicht das, wovon dies die genaue Kopie ist? was mit diesen bestimmten Worten zu beschreiben wäre? Hätte es also Sinn, zu sagen, es enthalte jene Figur jetzt? oder habe sie enthalten? - Die Bemerkung ist also zeitlos und man kann sie "geometrisch" nennen. ${ }^{19}$

Aspekte sind also logische, begriffliche oder geometrische Zusammenhänge, die wir am Gesehenen entdecken können oder in die wir das Gesehene stellen im Sinne einer Interpretation. Aspekte entsprechen Eigenschaften am Objekt, die auf anderen, schon bekannten Eigenschaften aufbauen. Sprachlich drücken wir Aspektwahrnehmung oft dadurch aus, dass wir von einem "Bemerken « und nicht von einem "Sehen" sprechen. Wir sehen das Vexierbild und bemerken plötzlich die

18 Siehe PUII S. 549. Siehe auch BPP $3 \$ 510$. Wittgenstein nennt interne Relationen manchmal einen "Zug", im Sinne von Gesichtszügen (siehe schon Tractatus 4.1221).

19 BPP3 $\$ 146$. 
Linie, die sich darin verbirgt. Wir sehen die beiden Gesichter und bemerken dann eine Ähnlichkeit in ihnen.

\section{IV}

Wie lässt sich nun unsere visuelle Wahrnehmung beim Bemerken von Aspekten erklären? Man könnte folgendermaßen argumentieren: Auf einer ersten visuellen Ebene sind uns Eindrücke gegeben, die eine Art Beschreibung des Gesehenen wiedergeben. Man sieht zum Beispiel die Formen und Farben eines Gegenstandes, so dass man sie und ihre Veränderungen beschreiben könnte. Auf einer zweiten Ebene findet dann eine Interpretation des Gesehenen statt. Es gibt Regeln und Deutungen, die auf der ursprünglichen Wahrnehmung aufbauen, so dass wir unsere Schlüsse ziehen. Nur die erste Ebene ist wirklich ein Sehen, während die zweite Ebene eine Interpretation des Gesehenen wäre, die nicht mit visuellen, sondern mit kognitiven Begriffen zu erklären wäre, also mit Begriffen wie auffassen, deuten, folgern et cetera.

Am Beispiel der verschiedenen Aspekte des Dreiecks kann dieses Modell leicht verdeutlicht werden. Die Form des Dreiecks kann gesehen werden und man kann davon eine genaue Beschreibung geben - zum Beispiel mit Hilfe eines Koordinatensystems. In einer solchen Beschreibung bliebe jedoch unerwähnt, in welche Richtung das gesehene Dreieck zeigt oder orientiert ist. Das Zeigen wäre eine Frage der Deutung oder der Auffassung und nicht des Sehens. Man sieht das Dreieck und versteht es dann als etwas, das in verschiedene Richtungen zeigt - das eine ist ein visuelles Erlebnis, das andere ein kognitives. Doch die Beispiele der Aspektwahrnehmung widersetzen sich einem solchen Zwei-Stufen-Modell visueller Wahrnehmung. Denn man interpretiert das Dreieck oder den Hasen-Enten-Kopf eben nicht nur unterschiedlich, sondern man sieht sie auch anders. Die Figuren sehen anders aus, wenn man sie visuell "umklappen« lässt. Aspektsehen zeigt uns also, dass man Dinge nicht nur verschieden deuten, sondern gemäß ihrer Deutung auch verschieden sehen kann. ${ }^{20}$ Die Interpretation ist hier Teil der visuellen Wahrnehmung.

Der naheliegende Schluss wäre, den Umfang der möglichen visuellen Eindrücke zu erweitern. Vertreter der Gestaltpsychologie zum Beispiel argumentieren, dass unsere Seherlebnisse nicht nur Farb- und Form-Qualitäten haben, sondern auch »Gestalt-Qualitäten«. Wir sehen nicht nur Formen und Farben, sondern auch Gestalten, Orientierungen, Organisationen et cetera. Die Argumentation von Wolfgang Köhler, ${ }^{21}$ einem Hauptvertreter der Gestaltpsychologie, ließe sich am Beispiel unseres Dreiecks wie folgt zusammenfassen: Die Wahrnehmung des

20 Siehe BPP1 $\$ 2$; PUII S. 530d.

21 Wolfgang Köhler: Gestalt Psychology, New York 1929. Wittgenstein verweist in BPP1-3 mehrmals auf ihn. 
Dreiecks hinsichtlich seiner kopierbaren Form und Farbe bleibt offenbar unverändert, wenn wir das Dreieck in seinen beiden verschiedenen Aspekten sehen. Weil der visuelle Eindruck sich aber dabei ändert, muss es eben noch andere visuelle Qualitäten geben. Unsere Wahrnehmung des Dreiecks hat also nicht nur Form- und Farbqualitäten, sondern auch "Gestaltqualitäten" wie zum Beispiel die visuell erlebte Orientierung des Dreiecks. Die Wahrnehmung einer Gestalt entspricht einem zusätzlichen visuellen Eindruck.

Für Wittgenstein ist eine solche Erklärung jedoch problematisch, insbesondere wegen der schon erwähnten kategorialen Unterschiede. Visuelle Wahrnehmung und insbesondere visuelle Eindrücke sind Zustände, die einer empirischen Beschreibung des Gesehenen entsprechen. Durch sie erkennen wir physische Eigenschaften der gesehenen Objekte. Interpretationen oder Deutungen entsprechen dagegen regelhaften oder logischen Zusammenhängen, die einem gesehenen Objekt auf Grund von begrifflichen Beziehungen oder durch seine Verwendungsweise zueigen sind. Es wäre missverständlich, solche Zusammenhänge oder Interpretationen einfach mit zusätzlichen visuellen Eindrücken in Verbindung zu bringen. Denn dadurch würde suggeriert, dass sie unabhängig von den anderen visuellen Eindrücken stattfinden können. Es wäre zum Beispiel möglich, den visuellen Eindruck einer Ähnlichkeit zu haben, ohne die jeweiligen Objekte selbst zu sehen. Das widerspricht jedoch nicht nur unserer Erfahrung, sondern auch dem Begriff der Ähnlichkeit. Die visuelle Wahrnehmung von Ähnlichkeit und insgesamt von Aspekten, ist kein einfacher Zusatz zur übrigen visuellen Wahrnehmung, sondern baut darauf auf und ist somit strukturell verbunden. Das eine kann nicht ohne das andere stattfinden. Wenn visuelle Wahrnehmung als eine Ansammlung von Eindrücken verstanden wird, dann gerät sie durch eine Ergänzung zum »seltsam schwankenden Gebilde«, ${ }^{22}$ wie Wittgenstein es formuliert.

Wie aber ist die Wahrnehmung von Aspekten dann zu erklären? Wittgensteins Antwort ist radikal. Wenn wir einen neuen Aspekt sehen, dann haben wir keine zusätzlichen visuellen Eindrücke, sondern gesamte der Rahmen, in dem die Eindrücke stattfinden, verändert sich. Nicht der Inhalt unserer Wahrnehmung ändert sich also, sondern die Form der Wahrnehmung. Nicht, was wahrgenommen wird, ändert sich, sondern wie es wahrgenommen wird. Verschiedene Aspekte, die wir sehen, entsprechen verschiedenen Begriffen visueller Wahrnehmung und nicht verschiedenen Eindrücken innerhalb dieser Begriffe.

Diese These bedarf einer Erklärung. Wittgensteins Ausgangspunkt ist die Abhängigkeit zwischen unserer visuellen Wahrnehmung einerseits und der Beschreibung oder Darstellung ${ }^{23}$ der dabei gesehenen Dinge andererseits. Denn es gilt zweierlei: Die Art und Weise, wie wir Dinge sehen, hat einen Einfluss darauf, wie wir diese Dinge beschreiben können. Wenn wir Farben und Formen eines Gegenstandes

22 PUII S. 523d.

23 Darstellungen sind bei Wittgenstein eine weiter gefasste Form von Beschreibungen. Sie sind nicht an das Medium der Sprache gebunden und können auch zum Beispiel eine Zeichnung des Gesehenen sein. 
(bei gutem Licht) sehen können, dann können wir die gesehenen Gegenstände auch so beschreiben. Aber umgekehrt beurteilen wir auch unsere visuelle Wahrnehmung nach den Beschreibungsweisen, die sie uns ermöglicht. Wir sprechen von einem Farb- oder Formsehen, weil uns dabei eine Beschreibung von Formen und Farben ermöglicht wird. "Was ist das Kriterium des Seherlebnisses? - was soll das Kriterium sein? Die Darstellung dessen 'was gesehen wird ‘ ${ }^{24}$ schreibt Wittgenstein. Die Fähigkeit, gesehene Dinge auf eine bestimmte Weise zu beschreiben oder darzustellen, ist nicht nur der Schlüssel, sondern die ganze ontologische Grundlage für die visuelle Wahrnehmung. ${ }^{25}$

Doch es gibt sehr viele verschiedene Arten und Weisen zu beschreiben, was wir sehen, so Wittgenstein weiter. Und somit ist auch unser Begriff von visueller Wahrnehmung nicht einheitlich. ${ }^{26}$ Man kann visuelle Wahrnehmung beschreiben, indem man die gesehenen Formen und Farben mit Hilfe eines Bildes angibt. ${ }^{27}$ Dementsprechend stellen wir uns die visuellen Eindrücke als eine Art Ebene vor. Wir fassen die Wahrnehmung als ein »inneres Bild « auf. ${ }^{28}$ Ein solches inneres Bild erklärt die wesentlichen Elemente des Sehens recht genau: Es zeigt, dass wir Farben und Formen erkennen können und dass hintereinander liegende Gegenstände sich verdecken können. Gleichzeitig wird deutlich, was man nicht direkt sehen kann: Wenn eine Eigenschaft keine Auswirkung auf die Farbe oder Form eines Gegenstandes hat, dann kann sie auch nicht gesehen werden. Wir sehen weder die Temperatur eines Körpers noch sein Gewicht. Beides könnte zwar Teil einer sprachlichen oder grafischen Darstellung des Gegenstandes sein, aber das »innere" Bild, das man sich in etwa wie eine Fotografie vorstellt, bleibt davon unberührt.

Doch wie die Beispiele der Aspektwahrnehmung gezeigt haben, gibt es visuelle Eindrücke, die nicht der Farbverteilung auf einem solchen »inneren Bild « entsprechen. Wir können die gleiche Figur unterschiedlich sehen, obwohl sie nur einem Bild entspricht. Aber auch der räumliche Eindruck visueller Wahrnehmung wird durch ein »inneres Bild « nicht vollständig erfasst. Manche Bilder sind mehrdeutig hinsichtlich der dritten Dimension und außerdem sieht ein dreidimensionaler Gegenstand oder ein Hologramm wegen unserer Fähigkeit zum Stereosehen "räumlicher« aus als jedes flache Bild. Räumliches Sehen wird besser durch ein

24 PUII S. 526c; siehe auch 526e.

25 Wittgensteins Redeweise von »Kriterien« macht deutlich, dass der Zusammenhang "grammatisch « verstanden werden muss. Erst im Zusammenhang mit einer bestimmten Darstellungsweise im Sprachspiel gibt es das jeweilige Seherlebnis.

26 "Der Begriff der Darstellung des Gesehenen, sowie der Kopie, ist sehr dehnbar, und mit ihm der Begriff des Gesehenen." PUII S. 526d.

27 Den gewöhnlichen Begriff eines visuellen Erlebnisses hält Wittgenstein tatsächlich dem eines inneren Bildes vergleichbar BPP3 $\$ 513$; BPP1 $\$ 896$ oder Ms 133 S. 88.

28 Diese Auffassung findet sich häufig bei Vertretern einer Sinnesdaten-Theorie und wird auch mit dem Retina-Bild in Verbindung gebracht. Für eine mehr begriffliche Rekonstruktion als "visuelles Feld" siehe Christopher Peacocke: Sense and Content. Experience Thought and Their Relations, Oxford 1983. 
räumliches Modell der gesehenen Dinge beschrieben als durch ein Bild. Wir ändern also die Darstellungsweise und verwenden statt des flachen Bildes eine dreidimensionale Figur, um das Gesehene zu beschreiben. Wichtig zu erkennen ist nun der begriffliche Unterschied zwischen diesen beiden Darstellungsweisen. Die räumliche Darstellung erhält man nicht, indem man dem flachen Bild zusätzliche Qualitäten hinzufügt. In der räumlichen Darstellung gibt es das flache Bild gar nicht mehr. Die Beschreibung durch das Bild wird transformiert und nicht ergänzt. Und dieser Unterschied überträgt sich auch auf die visuelle Wahrnehmung, die ja mit den verschiedenen Darstellungsweisen verbunden ist. Der Unterschied zwischen einer Wahrnehmung ohne räumliche Tiefe (zum Beispiel wenn man ein Auge schließt) und einer visuellen Wahrnehmung mit räumlicher Tiefe, ist vor allem ein Unterschied zwischen zwei Begrifflichkeiten und nicht zwischen zwei Eindrücken. ${ }^{29}$ Es sind jeweils verschiedene Formen der Darstellung des Geschehenen angemessen.

Dies ist also Wittgensteins Idee: Ein Betrachter, der einen Aspekt sieht, zum Beispiel wenn er die räumliche Dimension eines Gegenstandes erkennt, eine Ähnlichkeit sieht oder eine Figur plötzlich als Entenkopf wahrnimmt, dessen Wahrnehmung verändert sich in dem Sinne, dass er das Gesehene neu darstellen und mit neuen Begriffen beschreiben kann. Aber weil unsere visuelle Wahrnehmung nichts anderes ist, als der Ausgangspunkt einer solchen Beschreibungsweise, verändert sich damit unsere Vorstellung der visuellen Wahrnehmung selbst. Den Hasen-Enten-Kopf als Hase oder Ente zu sehen bedeutet nichts anderes als ihn einmal als Hasen und einmal als Ente beschreiben zu können. Ein Wechsel beziehungsweise ein Unterschied zwischen zwei gesehenen Aspekten entspricht daher einem Wechsel beziehungsweise einem Unterschied zwischen zwei verschiedenen Darstellungsweisen des Gesehenen. Damit bleibt der kategoriale Unterschied zwischen dem Sehen von empirischen Eigenschaften und dem Sehen (Bemerken) eines logisch-begrifflichen Zusammenhangs erhalten. Denn beim Übergang von einer Beschreibung zu einer anderen erhalten wir keine neue empirische Aussage über einen Gegenstand, sondern eine neue Interpretation. Das Sehen eines Aspekts kann folglich auf zwei verschiedene Weisen aufgefasst werden. Entweder als ein Sehen des Aspektes innerhalb einer bestimmten Darstellungsweise. Oder aber als eine Interpretation des Gesehenen, wenn man diese Darstellungsweise ins Verhältnis zu einer ursprünglichen oder einfach verschiedenen visuellen Wahrnehmung und ihrer Darstellungsweise setzt. Wer plötzlich eine Ähnlichkeit in zwei Gesichtern bemerkt, der sieht die Gesichter als ähnlich - Ähnlichkeit ist eine Eigenschaft, die man auch sehen kann. Aber gleichzeitig handelt es sich um eine Interpretation der ursprünglichen visuellen Wahrnehmung.

Dieser Doppelstatus macht Aspektsehen aus. Man kann es nicht aufteilen in ein visuelles Erlebnis einerseits und einen kognitiven Vorgang andererseits. Aspektsehen ist keine Mischung aus Sehen und Interpretieren, sondern es befindet sich

29 BPP1 $\$ 85$. 
begrifflich dazwischen. Es »kreuzen« sich verschiedene Begriffe. ${ }^{30}$ Sehen von Aspekten hat einerseits Gemeinsamkeiten mit unseren Begriffen von einem Auffassen, Interpretieren, Denken oder Verstehen und andererseits Gemeinsamkeiten mit unserem Begriff von einem Sehen im Sinne einer Wahrnehmung von Formen und Farben. Es kann nicht dem einen oder anderen zugeordnet werden, sondern es gibt Gemeinsamkeiten mit beiden: „Es ist ein Sehen, insofern ... Es ist ein Sehen nur insofern, als ... (Das scheint mir die Lösung.) «31 schreibt Wittgenstein.

\section{V}

Kehren wir nun zurück zu der Wahrnehmung visueller Modelle und zu unserem Beispiel des Modellwirbels. Die Frage war, auf welche Weise wir die Bewegungsrichtung der Linien sehen können oder gar den Wirbel selbst in den Linien, die doch nur eine starre Form bilden. Wenn Wittgensteins Analyse zutrifft, dann handelt es sich dabei um die Wahrnehmung von visuellen Aspekten des Modells. So wie wir die Orientierung eines Dreiecks sehen können, so können wir auch die Richtung der "Illuminated Field Lines" sehen. So wie wir eine Figur als einen Hasenkopf sehen können, so können wir die Linien als einen Wirbel sehen. Unsere Wahrnehmung von visuellen Modellen ist nicht nur die Wahrnehmung von visuellen Eigenschaften des Modells, die anschließend auf etwas Externes übertragen werden. Sondern es werden dabei Aspekte wahrgenommen, in denen diese Interpretation schon enthalten ist.

Wittgensteins Analyse eröffnet somit eine neue Perspektive auf die Anschaulichkeit von visuellen Artefakten im Allgemeinen und von visuellen Modellen im Speziellen. Modelle sind nicht nur vereinfachte oder idealisierte Illustrationen von Erkenntnissen, die sprachlich oder theoretisch formuliert werden können. Um sie zu verstehen, müssen wir sie nicht in ein »echtes" Wissen übersetzen, sondern in ihrer Visualität ist ein Teil des Verstehes der modellierten Dinge schon enthalten. Indem wir ein visuelles Modell sehen, verstehen wir etwas über das Modellierte. Die visuelle Wahrnehmung beinhaltet einen Teil der Erklärung, die das Modell uns gibt.

Das Verständnis, das durch visuelle Modelle vermittelt wird, unterliegt dann jedoch ebenfalls einer Neubewertung. Es handelt sich um ein Verständnis, das essentiell mit visueller Wahrnehmung verknüpft ist und nicht davon getrennt werden kann. Einen Luftwirbel, eine Dampfmaschine oder ein architektonisches Konzept zu verstehen bedeutet nicht nur, sprachlich formulierbares Wissen zu gewinnen oder Theorien darüber zu erwerben, sondern es kann auch darin bestehen, dass man sich "ein Bild" von den Dingen macht. Dieses dezidiert visuelle Verständnis ermöglicht uns einen eigenen epistemischen Zugang zu den Dingen.

30 PUII S. 549a.

31 BPP2 $\$ 390$. Siehe auch PUII S. 537c, d und BPP2 $\$ 462$. 
Es ist die Fähigkeit, visuelle Phänomene in verschiedenen Situationen wiedererkennen zu können, spontan auf Veränderungen reagieren zu können oder Dinge als etwas Bestimmtes aufzufassen und beschreiben zu können - Fähigkeiten, die eng mit dem Begriff des Sehens verbunden sind. Die Leistung von visuellen Modellen ist dafür unersetzlich. So wie Worte die Träger für ein sprachliches Verständnis sind, so sind visuelle Modelle die Träger für ein eigenes visuelles Verständnis, das sie in Form von Aspekten inkorporieren. Visuelle Modelle eröffnen uns damit einen Zugang zur Welt, der oftmals die Voraussetzung dafür bildet, sich überhaupt sprachlich mit ihr auseinanderzusetzen. 
Ingeborg Reichle, Steffen Siegel, Achim Spelten (Hg.)

Visuelle Modelle

Wilhelm Fink 


\section{INHALT}

Ingeborg Reichle, Steffen Siegel, Achim Spelten

Die Wirklichkeit visueller Modelle ........................ 9

\section{Begriff und Metapher}

Bernd Mahr

Cargo.

Zum Verhältnis von Bild und Modell

Achim Spelten

Visuelle Aspekte von Modellen

Samuel Strehle

Evidenzkraft und Beherrschungsmacht.

Bildwissenschaftliche und soziologische Zugänge

zur Modellfunktion von Bildern $\ldots \ldots \ldots \ldots \ldots \ldots \ldots \ldots \ldots \ldots \ldots \ldots \ldots \ldots \ldots \ldots \ldots$

Tobias Schlechtriemen

Metaphern als Modelle.

Zur Organismus-Metaphorik in der Soziologie ................. 71

Philipp Ekardt

Benjamins Bekleidungsmodelle.

Strumpf und Rüsche als Topologien der Bildtheorie .............. 85

\section{EXPERIMENT UND Wissen}

ReINHARD WendLeR

Das Spiel mit Modellen.

Eine methodische Verwandtschaft künstlerischer Werk-

und molekularbiologischer Erkenntnisprozesse 
Sebastian Vincent Grevsmühl

Das modellierte Antlitz der Erde.

Zur Geschichte der Modellierungsstrategien

der Kontinentalverschiebung . .

Carolin Artz

Das Fotogramm als visuelles Modell?

Die Visualisierung nichtsichtbarer Strahlen

in wissenschaftlichen Fotografien um 1900

INGEBORg REICHLE

Lebendige Kunst oder Biologische Plastik?

Reiner Maria Matysiks Prototypenmodelle

postevolutionärer Organismen

FARbTAFELN

\section{MAß UND RAUM}

Catharina Manchanda

Modelle und Prototypen.

Ein Überblick ........................................... 179

Steffen Siegel

Modell-Räume.

Architektur, Photographie, Topoklasmus ...................... 197

Der Blick ins Modell

Ein Gespräch mit Damaris Odenbach ....................... 215

Katrin Käthe Wenzel

Brot und Bauten.

Drei künstlerische Experimente zu

verformbarer Architektur

Stefan Riekeles

Ikodynamische Kreuzfahrt.

Sichtbarkeit und Tarnung in einer Arbeit

von Knowbotic Research 


\section{Zeit und Struktur}

Annemieke R. Verboon

Einen alten Baum verpflanzt man nicht.

Die Metapher des Porphyrianischen

Baums im Mittelalter ................................. 251

Sebastian Gießmann

Graphen können alles.

Visuelle Modellierung und Netzwerktheorie vor 1900 .............. 269

Jens Gulden

Semantik in visuellen Modellen.

Räumliche Regularitäten und körperliche

Erfahrungsmuster als Bedeutungsträger visueller Modelle ............ 285

Inge Hinterwaldner

Simulationsmodelle.

Zur Verhältnisbestimmung von Modellierung und

Bildgebung in interaktiven Echtzeitsimulationen

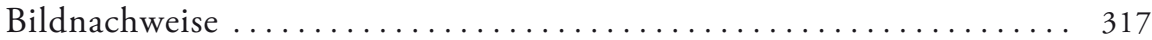

Autorinnen und Autoren ................................ 319 